\title{
Noms propres de territoires algériens : lieux de revendications et de conflits géoculturels, sociohistoriques et linguistiques
}

\begin{abstract}
:
The power of the name is recognized in all societies. Communities that it describes as "primitive" make use in the rituals of magic, but also to define their hunting territories. In the so-called "modern" societies, things have hardly changed: the name is also used to exercise a power to the individual; at the community level, it was decided to give to sites where they are appreciated by the political project of the dominant group names. He does not hesitate to change the names of places for a world as their beliefs and its prospects. This change is driven by historical, psychological and linguistic factors. Thus, a primary function of designation, the toponym has been graft a symbolic function that reflects the dominant group projects.

Key words: toponym, Algeria, France, change, adaptation, translation, language, multilingualism, history, territory
\end{abstract}

\section{Dr. Ahmed BOUALILI}

Département des lettres et langue française

Université Mouloud MAMMERI, Tizi-Ouzou

Chercheur associé CRASC

\section{Introduction :}

إن سلطة الأسماء معترف بها في كل المجتمعات، إذ في

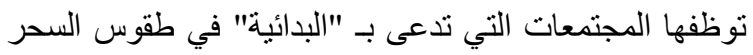

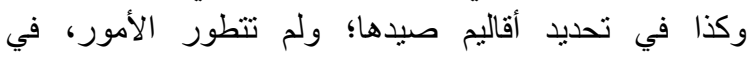

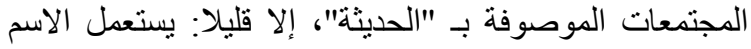

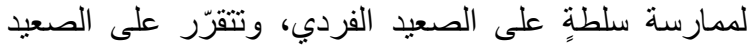

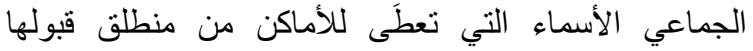

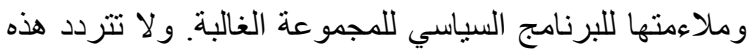

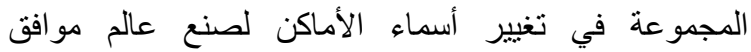

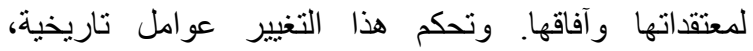
وسوسيونفسية ولغوية. من وظيفة التحديد الأولية تطعّم اسم اسم

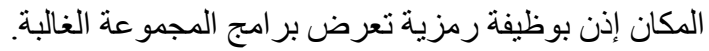

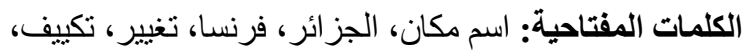
ترجمة، لغة، تعددية لغوية، تاريخ، إقليم.
L'État et la société exercent une influence, voire un contrôle, sur la toponymie engendrant une tension aux niveaux culturel, social et politique. Cette influence ou action sur la toponymie s'inscrit dans la «normalisation» toponymique.

L'uniformisation de l'écriture des toponymes a été suggérée dès le premier congrès international de géographie en 1871. En 1875 , lors du $2^{\mathrm{e}}$ congrès, on en précisait les recommandations.

Comme «le nom de lieu identifie, il localise, il délimite, il décrit, il caractérise, il rappelle, il perpétue, il évoque, il distingue ; souvent il hiérarchise et il structure : 
en un sens il approprie ${ }^{(1)} \gg$, l'État français, va appliquer ces recommandations en France métropolitaine mais aussi dans les colonies. C'est ainsi qu'en Algérie les noms autochtones vont être adaptés, modifiés, remplacés au gré du colonisateur.

En outre, le nom sert, entre autres, à revendiquer une place dans la société et c'est souvent celle du dominant car, en nommant, celui-ci exerce un pouvoir sur les choses. Ce pouvoir peut s'exercer sur l'espace qui devient territoire. ${ }^{(2)}$

C'est à juste titre que Bourdieu affirme que «l'espace est un des lieux où le pouvoir s'affirme ets'exerce, et sans doute sous la forme la plus subtile, cellede la violence symbolique, comme violence inaperçue $\left.{ }^{(3)}\right)$.

Durant l'histoire de l'Algérie des territoires ont été nommés et renommés au gré des occupants (Arabes et/ou Français). À titre d'exemple, une localité dans la région de Bouira (à l'Est d'Alger) était désignée avant l'arrivée des Français Imechdallen (fourmis rouges en berbère). Les Français l'ont appelée Mayot, alors que l'arabisation opérée après l'indépendance (1962), au lieu de réhabiliter le nom berbère, l'a baptisée Mechdallah (celui qui tient à Dieu).

Ce que nous proposons de faire à travers cette étude, c'est de reconstituer l'histoire de certains territoires dans leurs dénominations plurilingues (berbère, arabe et française) et voir comment cet acte de désignation est sous-tendu par des rapports de pouvoir et de réappropriation. Nous tenterons également de comprendre les représentations des locuteurs utilisant des noms (lesquels ? ceux d'avant ou ceux d'aujourd'hui) pour désigner ces territoires.

Notre travail se présentera en deux parties. Dans la première nous décrirons les changements dans les dénominations. Dans la seconde nous expliquerons les causes de ces changements.

Mais au préalable situons la problématique algérienne de la dénomination dans la situation mondiale de la toponymie. Un rappel des dispositions légales en matière d'onomastique semble nécessaire.

\section{Dispositions légales}

La Convention-cadre pour la protection des minorités nationales élaborée par le Conseil de l'Europe en 1995 prévoit à l'article 11, alinéa 3, de protéger «les dénominations traditionnelles locales, les noms de rues et autres indications topographiques destinées au public... dans la langue minoritaire ${ }^{(4)} \gg$.

La déclaration universelle des droits linguistiques adoptée en 1996 à Barcelone stipule :

Section III Onomastique

Article 31 - Toute communauté linguistique a le droit de préserver et d'utiliser dans tous les domaines et occasions son système onomastique.

Article 32. 1. Toute communauté linguistique a le droit de faire usage des toponymes dans la langue propre du territoire, tant en 
ce qui concerne les usages oraux et écrits que dans les domaines privés, publics et officiels.

Article 32. 2. Toute communauté linguistique a le droit d'établir, de préserver et de réviser la toponymie autochtone, et celleci ne peut être supprimée, altérée ou adaptée arbitrairement. Elle ne peut non plus être remplacée en cas de changement de conjonctures politiques ou autres ${ }^{(5)}$.

Qu'en est-il des droits linguistiques en matière de toponymie en Algérie?

\section{Langues et territoire en Algérie}

La politique linguistique en Algérie a été pendant longtemps discriminatoire provocant des sentiments et des attitudes négatives des locuteurs vis-à-vis de leur langue maternelle. Le discours politique ne tient compte que de l'opposition langue nationale et langue étrangère. Cette opposition va exclure pendant longtemps les langues maternelles.

Cette exclusion va se manifester entre autres dansleur absence totale du domaine public : médias, école, dénomination, toponymie, etc. L'onomastique de façon générale et la toponymie en particulier vont refléter cette tendance à l'unilinguisme dans une volonté politique (administrative ?) d'arabiser par exemple les noms de personnes et les noms de lieux.

Il est prouvé que la langue et le territoire sont les fondements de toutes les idéologies nationalistes (féodales ?) notamment dans les ex-colonies françaises sous l'influence du jacobinisme. Il n'est pas donc étonnant de constater que l'appropriation ou l'expropriation passent par la (re)dénomination :

[...] les noms ont une influence décisive sur le développement d'une identité groupale et territoriale. On peut également dire que la dénomination des lieux est un instrument de contrôle social de l'espace par un groupe donné, et que d'une certaine façon, c'est cette appropriation dénominative de l'espace qui transforme ce dernier en territoire ${ }^{(6)}$.

Quel est donc le rapport entre les langues et le territoire en Algérie ? Avant de répondre à cette question, nous allons passer en revue le statut des langues et leur utilisation en Algérie. 


\section{Sphères d'utilisation des langues en Algérie}

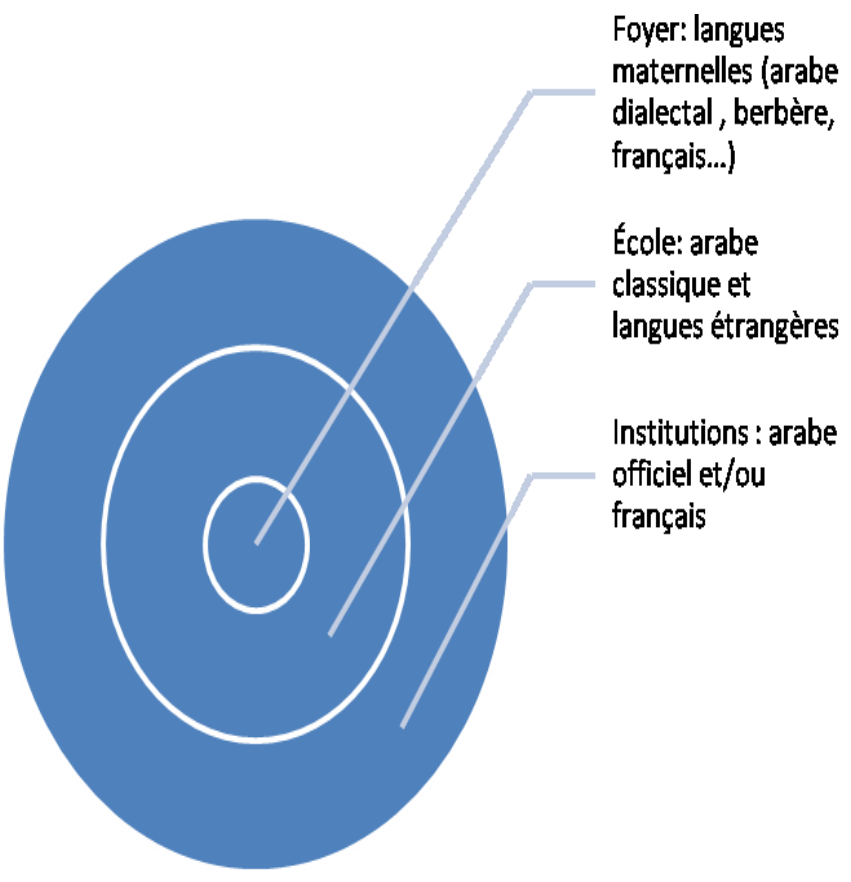

Modes d'utilisation des langues

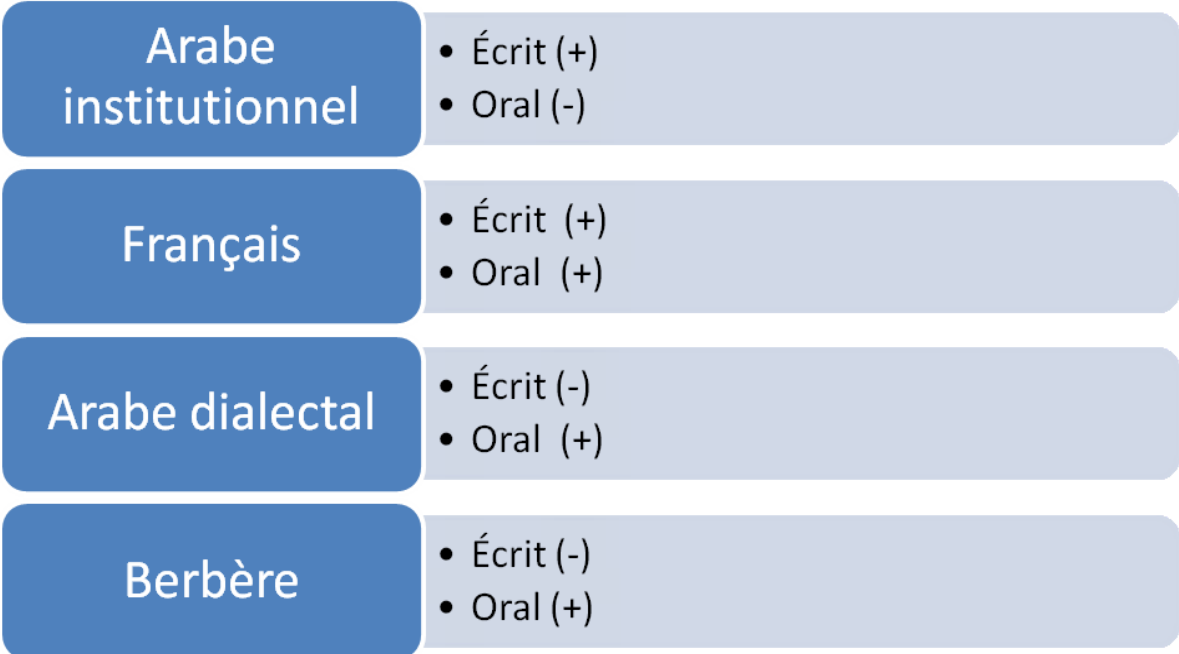

Il apparaît que la langue dont le mode d'utilisation est l'écrit et l'oral est le français. Quant aux autres langues, un mode est privilégié au détriment de l'autre. Cela explique en partie le statut privilégié dont bénéficie encore 
aujourd'hui le français en Algérie malgré les ressentiments laissés par l'histoire.

\section{Langues, dénomination et redénomination \\ Langues et dénomination}

L'Algérie est en situation de bilinguisme, même de plurilinguisme malgré l'apparence qu'on lui donne de pays monolingue. Cette étiquette est au service d'une idéologie unificatrice et utopique croyant à l'unification de la nation par l'unicité de la langue.

En 1998, dans une énième tentative d'arabisation forcée, le gouvernement a imposé l'utilisation de la seule langue arabe dans les noms de lieux; conscient de la portée de cette manœuvre dans la mesure où

[...] le nom de lieu appartient à celui qui le crée et à celui qui l'utilise ; il appartient aussi à celui qui possède ou qui fréquente le lieu ; il appartient, dans une certaine mesure, à celui qui le traite ; disons plutôt qu'il est à la merci de celui qui le traite. Car, en effet, "toucher" à un nom de lieu n'est jamais indifférent.»(Henri Dorion, 1990, p. 128)

l'Étatvoudrait, à travers cette opération, encore une fois, mettre fin à l'hégémonie du français dans la désignation populaire des lieux. Mais elle comptait également stopper l'élan revendicatif des minorités berbères qui voulaient un statut meilleur pour leurs langues notamment par la redénomination des lieux.

Dans les faits, cette injonction n'a pas fait long feu ; paradoxalement, elle a produit l'effet inverse, notamment en Kabylie ${ }^{(7)}$ où les noms de lieux transcrits en arabe ont été délibérément brouillés.

\section{La dénomination}

La dénomination est définie comme «activité de l'esprit humain, de caractère généralement collectif, qui a pour objet de mettre en relation un élément du réel et un signe du langage, donc de nommer. Cette activité, bien que présentant des caractéristiques universelles, se manifeste de façon fort différente suivant les collectivités $[\ldots]^{(8)}$ 》

Cela explique pourquoi les systèmes de dénominations sont si différents et pourquoi l'on procède à des redénominations, des remaniements, des adaptations, etc. jugeant le nom actuel inadapté ou inapproprié pour désigner le lieu.

La dénomination est à ce titre au cœur de l'activité toponymique qui d'une «pratique à l'origine "spontanée", anonyme, centrée sur la description des caractéristiques physiques, [...] a progressivement été pensée, systématisée et est devenue un instrument, une arme, un enjeu des politiques linguistiques $[\ldots]^{(9)_{\Downarrow}}$, voire à terme, une assimilation culturelle et linguistique. 
Ainsi, les tribus kabyles qui se désignaient par le filiatif $A t h$, d'origine kabyle, se sont vu appliquer une autre particule filiativebéni, qui, elle, est d'origine arabe.

\section{La redénomination}

La redénomination est, quant à elle, «l'ensemble des transformations affectant soit la forme soit le sens des dénominations premières [...] à savoir les différents modes de traduction, de (ré)interprétation ou de (re)motivation, sans oublier la substitution pure et simple $\mathrm{e}^{(10)} \gg$.

La redénomination est donc un processus linguistique qui affecte le signifiant d'un signe linguistique, à savoir le toponyme.

Celui-ci «retrace l'histoire du signifiant et cette histoire est ce qu'il apporte pour sa part aux représentants des autres disciplines. Le signifié reste sans doute intimement lié au signifiant : qu'est-ce qu'un signifiant qui ne déboucherait sur rien ? Le toponymiste ne l'ignore pas, mais sa tâche est de tirer du nom qu'il traite tous les renseignements qu'il peut $\left.(\ldots)^{(11)}\right\rangle$

Par ailleurs, de par sa définition, la toponymie est une science, quoique récente, qui se charge de l'étude des noms de lieux et de leurs transformations.Ernest Muret (Les noms de lieux dans les langues romanes) signalait déjà en 1930 l'appartenance linguistique du nom de lieu qui en ce sens est un mot comme les autres. Ce caractère fait que le toponyme est sujet aux changements notamment phonétiques.

Toutefois, il semblerait que le toponyme en particulier, et le nom propre en général, manifeste une certaine résistance à ces changements. Il est pourtant évident que le toponyme change ; mais cette transformation ne paraît pas "naturelle".

En effet, elle est souvent due à l'homme, mue par des facteurs sociologiques, psychologiques et historiques pour ne citer que ceux-là.

\section{Types de redénomination}

SalihAkin regroupe les redénominations dans trois "processus" majeurs :

« le premier processus est néologique et se traduit par la mise en circulation de nouvelles dénominations. Le deuxième processus se caractérise par la remise en circulation et la valorisation d'anciennes dénominations. Enfin, le dernier se manifeste par la remise en circulation d'anciennes dénominations assorties de procédés d'adaptation aux nouvelles donnes et normes linguistiques, politiques et sociales $^{(12)} . »$ 
Noms propres de territoires algériens : lieux de revendications et de conflits géoculturels, sociohistoriques et linguistiques

Le schéma suivant reprend dans le détail les différents types de redénomination signalé par SalihAkin plus haut. Il est certain que tous ces types ne se réalisent pas dans une seule situation linguistique. Une politique linguistique va en privilégier certains à d'autres pour des raisons diverses.

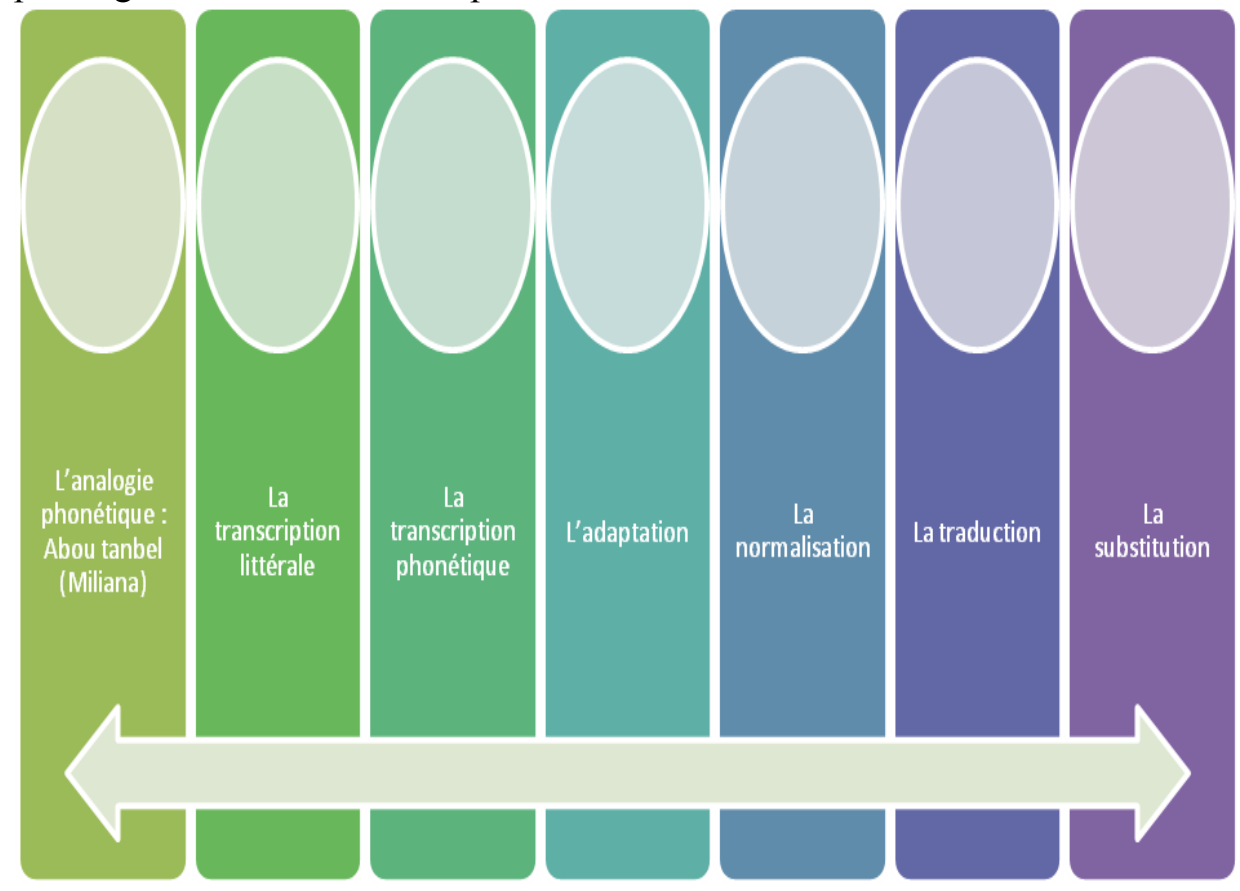

Redénomination en Algérie

Quelques exemples de redénominations ${ }^{(13)}$

\begin{tabular}{|l|l|l|l|}
\hline $\begin{array}{l}\text { Avant la } \\
\text { colonisation } \\
\text { (avant 1830) }\end{array}$ & $\begin{array}{l}\text { Durant la } \\
\text { colonisation } \\
(1830-1962)\end{array}$ & $\begin{array}{l}\text { Après } \\
\text { l'indépendance }\end{array}$ & $\begin{array}{l}\text { Type de } \\
\text { redénomination }\end{array}$ \\
\hline Oued Metnane & Montenotte & Sidi Akkacha & $\begin{array}{l}\text { Adaptation par } \\
\text { les français puis } \\
\text { substitution } \\
\text { après 1962 }\end{array}$ \\
\hline- & $\begin{array}{l}\text { On inscrit près } \\
\text { d'une source } \\
\text { "eau potable" }\end{array}$ & Abou Tanbal & $\begin{array}{l}\text { Analogie } \\
\text { phonétique }\end{array}$ \\
\hline
\end{tabular}




\begin{tabular}{|c|c|c|c|}
\hline Aïn El Berd & Oued Imbert & Aïn El Berd & $\begin{array}{l}\text { Analogie } \\
\text { phonétique }\end{array}$ \\
\hline AïnNouissy & Noisy-les-Bains & & $\begin{array}{l}\text { Transcription } \\
\text { littérale } \\
\text { traduction }\end{array}$ \\
\hline Ecosium, dzaïer & Alger & Djazaïr & $\begin{array}{l}\text { Substitution puis } \\
\text { transcription } \\
\text { littérale et enfin } \\
\text { adaptation }\end{array}$ \\
\hline - & Descartes & Ben Badis & Substitution \\
\hline Viscera & Biskra & Biskra & $\begin{array}{l}\text { Transcription } \\
\text { littérale }\end{array}$ \\
\hline Imechdallen & Maillot & Mechdallah & $\begin{array}{l}\text { Substitution puis } \\
\text { adaptation }\end{array}$ \\
\hline - & Rivet & Meftah & Substitution \\
\hline & Orléansville & $\begin{array}{l}\text { El Asnam } \\
=>\text { Chlef }\end{array}$ & Substitution \\
\hline \multicolumn{4}{|c|}{$\begin{array}{l}\text { Remarques } \\
\text { Il apparaît donc que le mode de redénomination le plus utilisé est la } \\
\text { substitution. La redénomination a plusieurs causes (accès à l'indépendance, } \\
\text { changement de régime, etc.). Lorsque le lieu porte un nom romain, les Français } \\
\text { ne procèdent pas à sa redénomination (Biskra, Tipasa, Sétif, etc.). } \\
\text { L'indépendance n'a pas pour autant rétabli les noms originels berbères ; on leur } \\
\text { préfère parfois les noms français avec ou sans adaptation (Tinuza=> La Calle } \\
\text { => El Kala). } \\
\text { Nous verrons dans ce qui suit les causes qui ont abouti aux changements } \\
\text { constatés. } \\
\text { Causes des redénominations } \\
\text { Les causes historiques } \\
\text { Comme l'attribution d'un non, d'un toponyme participe au processus } \\
\text { d'appropriation, la colonisation remplace les noms autochtones par des noms } \\
\text { français (BologhineibnouZiri => Saint-Eugène). }\end{array}$} \\
\hline
\end{tabular}


Partant des appellations des peuples, Calvet remarque que ce mépris «relève d'un mépris plus vaste pour les peuples; les territoires et les habitants n'existaient pas avant l'arrivée du colonisateur [...] et l'on nomme lieux et peuples comme bon nous semble ${ }^{(14)} . \gg($ Calvet, $2002: 81-82)$

L'indépendance,dans le souci de finir avec les traces de la colonisation,va paradoxalement faire appel au même processus redénominatif. Ainsi, un lieu portant le nom d'un "héros" français (Morris) va être remplacé par le nom d'un héros algérien (Ben Mehidi).

Le plus souvent, le nouvel État, par méconnaissance du nom originel ou par volonté délibérée de négation, procédera à la redénomination du lieu sans prise en compte des caractéristiques linguistiques régionales ou nationales; car en fin de compte

«l'idéologie nationale héritée de la révolution [...]

n'est en réalité que le miroir inversé du discours colonial sur l'Algérie [...]. L’idéologie coloniale travestissait l'histoire pour fonder la légitimité de la domination française. Le nationalisme lui empruntera sa démarche faisant à son tour bon marché du réel ${ }^{(15)} . »$

\section{Les causes politiques}

La France étant restée un siècle et demi en Algérie, le changement de régime en France métropolitaine a affecté les noms de lieu en Algérie. Ainsi Fort National est-il devenu Fort Napoléon sous le Second Empire.

Le changement de tendance politique en Algérie indépendante va apporter également son lot de redénominations. Sous le régime baathiste, prorusse et procommuniste, des noms référant au communisme vont apparaître un peu partout en Algérie.

L'exemple d'une place publique à Mostaganem (Ouest algérien) est édifiant. Jusqu'à la fin des années quatre-vingts, sous le régime baathiste, la grande place de la ville s'appelait la Place Rouge. Avec la montée de l'islamisme, cette place a été rebaptisée Place Verte, en référence à la couleur supposée du coran.

À la fin des années quatre-vingt-dix, pour accompagner la politique de réconciliation nationale qui visait à gracier les terroristes, des lieux vont porter des noms avec le complément déterminatif concorde civile. Ainsi la place du $1^{\text {er }}$ mai devient-elle la place de la concorde civile, le parc de Ben Aknoun est-il renommé le parc de la concorde nationale, etc.

\section{Les causes naturelles}

Comme on ne parle pas de corde dans la maison d'un pendu, nous avons relevé un changement dû à une cause naturelle. En 1980, se produit un séisme dévastateur dans une ville anciennement appelée Orléansville et renommée El 


\section{Dr. Ahmed BOUALILI}

Asnam (les ruines, en arabe) après l'indépendance en 1962. Ce nom ne plaisait plus aux habitants qui voulaient éloigner le souvenir du séisme. On décida alors de renommer encore une fois la ville ; on lui donna le nom actuel, Chlef.

\section{Les causes linguistiques}

L'idéologie nationaliste, "miroir inversé" de l'idéologie coloniale, a tenté de redorer le blason de la langue arabe une fois l'indépendance acquise. Pour ce faire, elle remplacera tous les noms français par des noms arabes.La reconquête du territoire va se faire encore une fois par la langue.

Dans la foulée, elle supprime les noms en langue berbère. L'acte de renommer procède de la volonté du colonisateur d'assimiler le colonisé. En nommant, le groupe le plus fort s'approprie le territoire et exerce un pouvoir sur l'objet nommé.

La politique française a été reconduite par le régime au pouvoir après l'indépendance. Les noms berbères de lieux ont été mis à l'écart au profit de noms arabes. Lorsque la tâche n'est pas facilitée par le colonisateur onremplace le nom français -, on opère une assimilation progressive (une partie du nom, celle qui est significative, est traduite: béni, oued, etc.)Pour ce faire, les gestionnaires des noms de lieuxuseront de la substitution, la traduction ou l'analogie phonétique.

Ainsi, «aux transformations à l'intérieur du même langage s'ajoutent, pour les noms de lieux comme pour les noms courants, celles qui résultent du passage d'une langue à une autre. Dans ce cas le nom est soit traduit, quand le sens est apparent (...), soit et c'est le cas le plus général - adapté à la langue emprunteuse ${ }^{(16)} . \gg$

Dans le cas de la substitution, des noms de lieux berbères vont être remplacés par des noms de lieux arabes: Temassinine, renommé par les Français Fort Flatters, puis Bordj Omar Dris, après l'indépendance.

Dans le cas de la traduction, la particule filiativeathdans tous les noms de lieux va être traduite en arabe. Ainsi Ath Douala devient-il Béni Douala. De même, le motassif(rivière en berbère) qui compose les hydronymes va être traduit en arabe par le motoued. (ex. AssifSibaou qui devient Oued Sibaou, etc.)

Dans le cas de l'analogie phonétique, le nom de lieu berbère Ighzer(ruisseau) va être renommé Isser puis Yasser, successivement durant la colonisation et après l'indépendance. Cette analogie phonétique a été parfois préjudiciable aux populations elles-mêmes. Pour l'anecdote, on a voulu renommer Rélizane (ancien nom français) en faisant une analogie phonétique; le nouveau nom donné est Ighillzane (le col des mouchesoule col de la merde, en berbère). Les gestionnaires se sont vite ravisés et lui préférèrent le nom "français".

Enfin, à la suite de SalihAkin, nous remarquons que même «si le changement du nom est essentiellement conditionné par des facteurs extralinguistiques 
(culturel, social, politique, historique), il s'inscrit aussi dans un fonctionnement normal de la langue, traversée par la variation linguistique ${ }^{(17) . »}$

\section{Attitudes des locuteurs}

Les résultats reproduits dans le tableau ci-dessous émanent d'enquêtes de terrain menées sous notre direction par des étudiants inscrits en magistère et en master ou effectuées par d'autres chercheurs. Elles ont été conduites dans des territoires arabophones, notamment Alger(18) et Médéa(19), et kabylophone, essentiellement Tizi-Ouzou ${ }^{(20)}$. Il s'agit d'enquêtes quantitatives dont l'objectif est d'étudier le rapport entre langue et dénomination en convoquant les variables de l'âge et de la langue maternelle. Il ressort de ces enquêtes ce qui suit :

\begin{tabular}{|c|c|c|}
\hline \multirow{2}{*}{$\begin{array}{l}\text { Génération de la } \\
\text { colonisation }\end{array}$} & \multicolumn{2}{|c|}{ Génération postindépendance } \\
\hline & Berbérophones & Arabophones \\
\hline $\begin{array}{l}\text { Utilise volontiers les } \\
\text { dénominations } \\
\text { françaises car ce sont } \\
\text { les noms qu'ils ont } \\
\text { assez souvent utilisé }\end{array}$ & $\begin{array}{l}\text { Partagés entre } \\
\text { nationalistes favorables } \\
\text { à l'arabisation } \\
\text { (révolutionnaire) des } \\
\text { noms de lieux et tenant } \\
\text { d'un discours identitaire } \\
\text { revendiquant la } \\
\text { renaissance de la } \\
\text { dénomination berbère }\end{array}$ & $\begin{array}{l}\text { Très influencés par } \\
\text { l'idéologie nationaliste } \\
\text { (une langue, un pays, } \\
\text { une nation) } \\
\text { Favorables à toutes les } \\
\text { redénominations sous } \\
\text { réserve qu'elles soient } \\
\text { en arabe }\end{array}$ \\
\hline
\end{tabular}

\section{Conclusion}

Selon Louis-Jean Calvet, «Tout commence par la nomination ${ }^{(21)}$ ». En réalité, tout commence et tout finit par la nomination. Dans les pays en "voie de conflit", l'acte de nomination est susceptible de changer d'acteur au gré des bouleversements politiques.

Seuls les traités internationaux peuvent garantir les droits des minorités à jouir de cet acte qui est toujours le même; seul le moyen de sa réalisation est différent. Ce moyen, c'est la langue du groupe fort du moment qui vise l'appropriation du territoire car le «droit de nommer est le versant linguistique du droit de s'approprier ${ }^{(22)}$ ».

Toutefois, le toponymiste ne doit pas céder facilement à la tentation de remonter le cours du temps en tentant de maintenir ou de bloquer l'évolution des toponymes qui sont somme toute des mots quelconques. Il doit tout au 
moins saisir les particularités linguistique, régionale, historique et sociopsychologique de ces mots.

En définitive, « on sait que le toponyme naît, vit, évolue, meurt et résiste comme un mot, mais on s'aperçoit que chaque cas renvoie à une particularité locale. On ne peut être ni trop "régionaliste", ni accepter le remplacement d'éléments locaux caractéristiques par des précisions inutiles ${ }^{(23)} 》$.

Bibliographie

Akin, Salih, «Pour une typologie des processus redénominatifs», dans Akin, Salih(dir.), Noms et Re-noms : La dénomination des personnes, des populations, des langues et des territoires, , Publications de l'université de Rouen-C.N.R.S, 1999,p.33-59

Bir, Fariza et Dahmani, Sarah, La toponymie et le marquage identitaire de l'espace dans la ville de Tizi-Ouzou, mémoire de master sous la direction de A. Boualili, Université Mouloud Mammeri de Tizi-Ouzou, 2015.

Bourdieu, Pierre, (dir.), La misère du monde, Paris, éd. du Seuil, 1993, 947 p.

Boussiga, Aissa, Marquage signalétique et appropriationde l'espace urbain : cas du centre-ville d'Alger, thèse de doctorat sous la direction de Rahal, Safia et Bulot, Thierry, Université d'Alger 2, 2014

Calvet,Louis-Jean, Linguistique et colonialisme, Paris, Ed. Payot et Rivages, 2002 [1974]

Chaurand, Jacques, «L'apport de la toponymie», dans Onomastique et histoire. Onomastique littéraire, Actes du VIIIe colloque de la Société Française d'Onomastique, Aix-en-Provence, 26-29 octobre 1994, Publications de l'université de Provence, 1998, 386 p.

Dorion, Henri, «L'apport de la recherche onomastique à la gestion des noms géographiques», dans Boulanger,Jean-Claude (dir.), Actes du XVIe Congrès International des Sciences Onomastiques, Québec, Université Laval, 16-22 août 1987, PUL, Québec 1990, p. 121-128, 591 p.

Falc'hun, François, «Le substrat celtique en Occitanie», dans Variation linguistique dans l'espace dialectologique et onomastique, Actes du XVIIe Congrès International de Linguistique et Philologie Romanes, Aix-enProvence, 29 août-3 septembre 1983, vol. 6, 1986, 645 p.

Guerrin, Christian, «Étude socio-toponymique des changements intervenus dans la nomenclature officielle des communes françaises entre 1943 et 1993», dans Onomastique et histoire. Onomastique littéraire, Actes du VIIIe colloque de la Société Française d'Onomastique, Aix-en-Provence, 26-29 octobre 1994, Publications de l'université de Provence, 1998, 386 p. 
Noms propres de territoires algériens : lieux de revendications et de conflits géoculturels, sociohistoriques et linguistiques

Guillorel,Hervé, «Toponymie politique», dans Akin,Salih(dir.), Noms et Re-noms : La dénomination des personnes, des populations, des langues et des territoires, sous la De, Publications de l'université de Rouen-C.N.R.S, 1999, p. 61-91.

Hamidi, Djamel, L'affichage publicitaire de la ville de Médéa, langue(s) et représentations, mémoire de magistère sous la direction de BoualiliAhmed, Université Mouloud Mammeri de Tizi-Ouzou, 2015.

Harbi, Mohamed, "Nationalisme algérien et identité berbère », dans Peuples Méditerranéens, $\mathrm{n}^{\circ} 11,1980, \mathrm{p} .31-37$.

Jonasson, Kirstin, Le nom propre : constructions et interprétations, Paris, Duculot, 1994, $255 \mathrm{p}$.

La déclaration universelle des droits linguistiques, Barcelone,1996; [en ligne]http://www.politique-africaine.com/numeros/pdf/064101.pdf, consulté le 25 juillet 2010.

Longnon, Auguste, Les noms de lieu de la France, leur origine, leur signification, leurs transformations, préf. de Jacques Chaurand, Paris, Honoré Champion, $831 \mathrm{p}$.

Malherbe, Michel, Quand l'histoire change les noms, les lieux à dénominations multiples, Paris, L'Harmattan,2008.

NdingaMbo, Abraham Constant, Onomastique et histoire au CongoBrazzaville, Paris, L'Harmattan, 2004, 228 p.

Plonéis, Jean-Marie, «La toponymie : mémoire des peuples», dans Onomastique et histoire. Onomastique littéraire, Actes du VIIIe colloque de la Société Française d'Onomastique, Aix-en-Provence, 26-29 octobre 1994, Publications de l'université de Provence, 1998, 386 p.

Ripoll, Fabrice et Vincent Veschambre, «Appropriation », ESO-UMR $6590, \mathrm{~N}^{\circ} 21$, mars 2004

Rostaing, Charles, Les noms de lieux, Paris, PUF, 1992 [1945], 126 p.

Villette, Guy, «La méthode en toponymie», In Cahiers de la Société française d'Onomastique, $\mathrm{N}^{\circ}$ 00, Dijon, automne 2008.

Annexe

Le corpus ${ }^{(24)}$

\begin{tabular}{|l|l|l|}
\hline Avant 1830 & De 1830 à 1962 & Après 1962 \\
\hline Oued Metnane & $\begin{array}{l}\text { Montenotte (Moutnote } \\
\text { à l'oral) }\end{array}$ & Sidi Akkacha \\
\hline & Cavaignac & Abou El Hassen \\
\hline & Boufice & Cinq-Palmiers \\
\hline & Henis & Bouzgaia \\
\hline & Rion-ville & Bnayria \\
\hline & Surcouf & Ain Chrob \\
\hline Aïn El Berd & Oued Imbert & Aïn El Berd \\
\hline
\end{tabular}


Dr. Ahmed BOUALILI

\begin{tabular}{|c|c|c|}
\hline & Michelet & Aïn El Hammam \\
\hline AïnNouissy & Noisy-les bains & AïnNouissy \\
\hline \multirow[t]{5}{*}{ Ecosium, dzaïer } & Alger & El Djazaïr \\
\hline & Colomb-Béchar & Béchar \\
\hline & Belcourt & Belouizdad \\
\hline & Descartes & Ben Badis \\
\hline & Morris & Ben Mehidi \\
\hline Vescera (antiquité) & Biskra & Biskra \\
\hline $\begin{array}{l}\text { Temassinine, } \\
\text { ZaouanTellouz } \\
\text { (Tamahaq) }\end{array}$ & Fort Flatters & Bordj Omar Dris \\
\hline \multirow[t]{2}{*}{ Ichariden } & $\begin{array}{l}\text { Fort National puis Fort } \\
\text { Napoléon sous le } \\
\text { Second Empire }\end{array}$ & LarbaaNathIrathen \\
\hline & Marengo & Hadjout \\
\hline Mina & Rélizane & IghilIzane \\
\hline Igilgilio (Antiquité) & Djidjeli & Jijel \\
\hline Ksar El Boukhari & Boghari & Ksar El Boukhari \\
\hline \multirow[t]{2}{*}{ Tinuza (berbère) } & La Calle & El Kala \\
\hline & Jeanne d'Arc & Larbi Ben Mehidi \\
\hline Imechdallen (berbère) & Maillot & Mechdallah \\
\hline \multirow[t]{3}{*}{ Lamida (romaine) } & Médéa & Médéa (lemdia) \\
\hline & Rivet & Meftah \\
\hline & Slissen & MoulaySlissen \\
\hline Laazib & Haussonvillers & Naciria \\
\hline \multirow[t]{4}{*}{ DjamaaGhezouah } & Nemours & Ghazaouet \\
\hline & Orléansville & $\begin{array}{l}\text { Al Asnam }=>\text { Chlef après } \\
\text { le séisme de } 1980\end{array}$ \\
\hline & Sainte-Barbe-du-Tlélat & Oued Tlélat \\
\hline & Warnier (ferni à l'oral) & OuledFares \\
\hline Bologhineibnouziri & Saint Eugène & Bologhine \\
\hline Siga & Saint-Denis-du-Sig & Sig \\
\hline Terga & Turgot & Terga \\
\hline Tagdempt & Tiaret & Tiaret \\
\hline Tipasa & Tipasa & Tipaza \\
\hline Tilimsen (berbère) & Tlemcen & Tlemcen \\
\hline \multirow[t]{4}{*}{ Yellel } & L’Hillil & Yellel \\
\hline & Eau potable & Abou tenbal \\
\hline & $\begin{array}{ll}\text { Place } & \text { rouge } \\
\text { (Mostaganem) }\end{array}$ & Place verte \\
\hline & Champ de Manœuvres & Place $\mathrm{du} 1^{\text {er }}$ mai $=>$ \\
\hline
\end{tabular}


Noms propres de territoires algériens : lieux de revendications et de conflits géoculturels, sociohistoriques et linguistiques

\begin{tabular}{|c|c|c|}
\hline & & $\begin{array}{l}\begin{array}{l}\text { place de la concorde } \\
\text { civile }\end{array} \\
\end{array}$ \\
\hline & Fort de l'eau & Bordj el Kiffan \\
\hline Ath Douala & Béni Douala & Béni Douala \\
\hline Ath Yanni & Béni Yanni & Béni Yanni \\
\hline Imezdhathen & La cité & La cité \\
\hline ThimizerBousmail & Le garage, le bataillon & Agarage, abatailloune \\
\hline Thaarkouvth & Le camp & Thaarkouvth \\
\hline TiziOuzou & Le village & Tiziouzou (bilage) \\
\hline Ath smail & & Sidi Abderrahman \\
\hline $\begin{array}{ll}\text { Iflissen } & \text { (berbère), } \\
\text { Draa Ben } & \text { Khedda } \\
\text { (ottoman) } & \end{array}$ & Mirabeau & DBK/Mirabeau \\
\hline Ighzer (berbère) & Isser & Yessar \\
\hline
\end{tabular}

\section{Notes :}

1-Henri Dorion, «L'apport de la recherche onomastique à la gestion des noms géographiques», dans Boulanger,Jean-Claude (dir.), Actes $d u$ XVIe Congrès International des Sciences Onomastiques, Québec, Université Laval, 16-22 août 1987, PUL, Québec 1990, 591 p., p. 128

2-«Le temps est révolu où le terme de territoire renvoyait surtout au juridique et au politique, à la frontière et à la loi, au pouvoir institutionnalisé. Il est aujourd'hui souvent défini sommairement comme un fragment d'espaceapproprié. », In Fabrice Ripoll et Vincent Veschambre, «Appropriation », ESO-UMR 6590, N 21, mars 2004,p.10

3-Pierre Bourdieu, 1993, La Misère du monde, p. 163

4-Hervé Guillorel, «Toponymie politique», dans Akin,Salih(dir.), Noms et Renoms : La dénomination des personnes, des populations, des langues et des territoires, sous la De, Publications de l'université de Rouen-C.N.R.S, 1999, p. 88

5-La déclaration universelle des droits linguistiques, Barcelone,1996; [en ligne]http://www.politique-africaine.com/numeros/pdf/064101.pdf, consulté le 25 juillet 2010.

6-HérvéGuillorel, op. cit., p. 63-64

7-Région montagneuse du Nord-Est de l'Algérie où est concentrée une population parlant le kabyle, une variété du berbère ou tamazight.

8-Mounin, Georges, (dir.), Dictionnaire de la linguistique, Paris, P.U.F., coll. Quadrige, 1974, p.99-100.

9-Hervé Guillorel, op. cit., p. 64 
10-Ibid., p. 72

11-Longnon, Auguste, Les noms de lieu de la France, leur origine, leur signification, leurs transformations, préf. de Jacques Chaurand, Paris, Honoré Champion, 1999 [1929], XII-XIV-831 p.

12-SalihAkin, «Pour une typologie des processus redénominatifs», dans Akin, Salih(dir.), Noms et Re-noms: La dénomination des personnes, des populations, des langues et des territoires, , Publications de l'université de Rouen-C.N.R.S, 1999, p. 36

13-Pour d'autres exemples, voir le tableau en annexe.

14-Louis-Jean Calvet, Linguistique et colonialisme, Paris, Ed. Payot et Rivages, 2002 [1974], p. 81-82

15-Mohamed Harbi, «Nationalisme algérien et identité berbère», dans Peuples Méditerranéens, $\mathrm{n}^{\circ} 11,1980$, p. 31-37, p. 31-32

16-Charles Rostaing, Les noms de lieux, Paris, PUF, 1992 [1945], 126 p., p. 20-21

17-SalihAkin, op. cit., p. 38

18-Nous signalons ici la thèse de Boussiga, Aissa, Marquage signalétiqueet appropriationde l'espace urbain : cas du centre-ville d'Alger, thèse de doctorat sous la direction de Rahal, Safia et Bulot, Thierry, ENS d'Alger, 2014

19-Cf. Hamidi, Djamel, L'affichage publicitaire de la ville de Médéa, langue(s) et représentations, mémoire de magistère sous la direction de A. Boualili, Université Mouloud Mammeri de Tizi-Ouzou, 2015.

20-Cf. Bir, Fariza et Dahmani, Sarah, La toponymie et le marquage identitaire de l'espace dans la ville de Tizi-Ouzou, mémoire de master sous la direction de A. Boualili, Université Mouloud Mammeri de Tizi-Ouzou, 2015.

21-Ibid., p. 80

22-Ibid., p. 82

23-Christian Guerrin, «Étude socio-toponymique des changements intervenus dans la nomenclature officielle des communes françaises entre 1943 et 1993», dans Onomastique et histoire. Onomastique littéraire, Actes du VIIIe colloque de la Société Française d'Onomastique, Aix-en-Provence, 26-29 octobre 1994, Publications de l'université de Provence, 1998, 386 p., p. 140

24-En partie tiré de Malherbe Michel,Quand l'histoire change les noms, les lieux à dénominations multiples, L'Harmattan, Paris, 2008. 